\title{
Respiratory complications in the early post-operative period following elective craniotomies
}

\author{
Sachidanand J. Bharati, Mihir P. Pandia', Girija P. Rath ${ }^{1}$, Parmod K. Bithal', Hari H. Dash ${ }^{2}$
}

\begin{abstract}
Background and Aims: Respiratory complications are of major concern after intracranial procedures. The objective of the study was to assess the incidence of respiratory complications in the initial 72 hours after elective craniotomies and to identify the associated risk factors. Materials and Methods: Patients undergoing elective craniotomies were studied prospectively. Information pertinent to history, physical examinations, investigation reports, perioperative events and outcome at discharge of the patients were recorded. Occurrence of any sign or symptom of respiratory system, need for reintubation/increased ventilatory support within 72 hours of surgery were considered as post-operative respiratory complication. Relationships of numerical variables and categorical variables with post-operative respiratory complications (PRCs) were assessed via T test and Chi-square (or Fisher's exact). Multivariate analysis using multiple logistic regression was performed for finding independent risk factors for respiratory complications. Results: Out of 96 I patients, I 37 (14.3\%) patients developed PRC within 72 hours of surgery. Ninety-nine (10.3\%) patients developed purulent tracheobronchitis. The patients who had PRC had longer hospital stays and poor Glasgow Outcome Scale at hospital discharge. The variables found as independent risk factors were tachycardia, blood transfusion in the intraoperative period and Glasgow Coma Scale (GCS) deterioration, hypokalemia and fever in the post-operative period. Conclusions: Respiratory complications within first 72 hours of elective craniotomies were common and were associated with prolonged hospital stay and poor neurological outcome. The variables which were found as independent risk factors were tachycardia, blood transfusion in the intraoperative period and GCS deterioration, hypokalemia and fever in the post-operative period.
\end{abstract}

Key words: Acute respiratory distress syndrome, craniotomy, intracranial procedures, pneumonia, respiratory complications, tracheobronchitis

\section{INTRODUCTION}

Post-operative respiratory complications (PRCs) in patients with intracranial lesions can lead to significant

\begin{tabular}{|l|l|}
\hline \multicolumn{2}{|c|}{ Access this article online } \\
\hline Quick Response Code: & Website: \\
\hline & www.jnaccjournal.org \\
\cline { 2 - 3 } & \\
\hline & \\
\hline
\end{tabular}

increase in the intensive care unit (ICU)/ hospital stay, morbidity and mortality. Assessment of the problem and identification of risk factors can be of great benefit for better management of these patients. There are only few studies that observed the occurrence of PRC in neurosurgical patients. ${ }^{[1-3]} \mathrm{We}$ did this prospective study to assess the respiratory complications occurring after craniotomy procedures and their effects on patient outcome. The objective of the study was to assess the incidence and characteristics of PRC in the initial 72 hours after craniotomy procedures and to identify the risk factors associated with these complications.

Department of Anaesthesia, Pain and Palliative Care, Dr. B. R. A. Institute Rotary Cancer Hospital, ${ }^{1}$ Department of Neuroanaesthesiology, All India Institute of Medical Sciences, ${ }^{2}$ Department of Anaesthesia, Fortis Memorial Research Institute, Gurgaon, New Delhi, India

Address for correspondence:

Dr. Mihir Prakash Pandia, Department of Neuroanaesthesiology, Room No. 709, Neurosciences Center, All India Institute of Medical Sciences, New Delhi - 110 029, India. E-mail: pandiamihir@gmail.com 


\section{MATERIALS AND METHODS}

After taking approval from the ethics committee of the institute, patients who would undergo elective intracranial procedures between August 2009 and October 2010 were included in the study. Using a standard proforma, the information pertinent to the history, physical examination, reports of investigations, premedication, anaesthesia technique, intraoperative events and post-operative complications were recorded. Patients were shifted to ICU in the post-operative period and subsequently to the neurosurgery ward. Occurrences of any respiratory complications in the first 72 hours of surgery were noted and patients were followed till discharge [Table 1].

Statistical analysis was done using software Statistical Package for the Social Sciences (SPSS)-15. Relationships

\section{Table 1: Definition of complication}

Respiratory complications: Post-operative respiratory complications included chest infection with purulent tracheobronchitis, pneumonia, ARDS, pulmonary oedema, pneumothorax, need for reintubation and tracheostomy. Occurrence of any of the signs or symptoms of respiratory system dysfunction like hypoventilation (respiratory rate $<8$ bpm), hypoxia (SpO2<90\%), airway obstruction, dyspnopea, bronchospasm, purulent tracheobroncitis (purulent tracheobronchitis was defined as fever, increased leukocyte count and increased tracheobronchial secretions with or without any chest infiltrates on X-ray) or the occurrence of atelectais, pneumonia (pneumonia was defined by fever, increased leukocyte count and increased tracheobronchial secretions with chest infiltrates on X-ray), pneumothorax, pleuraleffusion, ARDS(ARDSwas defined asfever,increased leukocyte count with bilateral chest infiltrates on X-ray and ratio of $\mathrm{Pao} 2 / \mathrm{Fio} 2<100$ ), respiratory failure (respiratory failure was defined as hypoventilation (respiratory rate $<8 \mathrm{bpm}$ ), hypoxia (SpO2<90\%) with laboured breathing requiring respiratory support), need for reintubation, tracheostomy or the need to increase ventilatory support were considered as respiratory complications

Cardiac complications: Change in blood pressure by $20 \%$ from baseline was defined as hypertension or hypotension. Heart rate of $>100 /$ minute was defined as tachycardia and $<50 /$ minute as bradycardia Occurrence of malignant hypertension, arrhythmias, myocardial ischaemia or any other cardiac complications requiring investigations and treatment were considered as cardiac complications

Neurological complications: Occurrence of delayed awakening, new motor or sensory deficit, cranial nerve palsies, seizures or deterioration of level of consciousness or other neurological complications requiring investigations and treatment were defined as neurological complications

Other complications: Other complications included post-operative fever (temperature $>100.4^{\circ} \mathrm{F}$ ), meningitis, electrolyte imbalances

ARDS = Acute respiratory distress syndrome of numerical variables and categorical variables with PRCs were assessed via T test and Chi-square (or Fisher's exact), respectively. For multivariate analysis, multiple logistic regression analysis was performed. The effects are shown as odds ratio (OR) with $95 \%$ confidence intervals (CI 95\%). $P$ values of less than 0.05 were considered as statistically significant.

\section{RESULTS}

The study sample included 961 patients who underwent elective neurosurgical procedure for intracranial pathologies. Demographic and perioperative characteristics are given in Tables 2-4. The mean age of the patients was 35 years, the youngest patient was 2 months and the eldest patient was 87 years old. Out of the 961 patients, $414(43 \%)$ patients were extubated following surgery and 547 (57\%) patients were electively ventilated in the ICU.

One hundred and thirty-seven (14.3\%) patients developed respiratory complications in the post-operative period [Table 5]. In the post-operative period, $68(7 \%)$ patients were reintubated in the first 72 hours after surgery.

Differences of outcome between patients with and without PRCs are given in Table 6. The overall mortality was $4.2 \%$. Poor outcome and hospital mortality was significantly high in patients with PRCs. The mean duration of hospital stay in patients who developed PRC was longer as compared to patients who did not have PRC [Table 6].

The variables which were found to be associated with development of post-operative respiratory complications were age, associated co-morbidities, preoperative cranial nerve palsies, preoperative motor deficits, position during surgery, haemodynamic disturbances (hypertension, hypotension, tachycardia), amount of blood loss, intraoperatve blood transfusion, duration of surgery, post-operative ventilation, post-operative neurological complications (Glasgow Coma Scale (GCS) deterioration, new neurological deficits, haematoma formation, cerebral infarct, post-operative hydrocephalus, generalized seizure, meningitis), electrolyte imbalances (hypokalemia, hypernatremia, hyponatremia), fever and diabetes insipidus (DI).

Factors which were found to be significant risk factors for occurrence of post-operative respiratory complications in the multivariate analysis were intraoperative tachycardia, intraoperative blood transfusion, GCS deterioration in the post-operative period, hypokalemia and fever in the post-operative period $(P<0.05)$ [Table 7]. 
Table 2: Preoperative factors and post-operative respiratory complications*

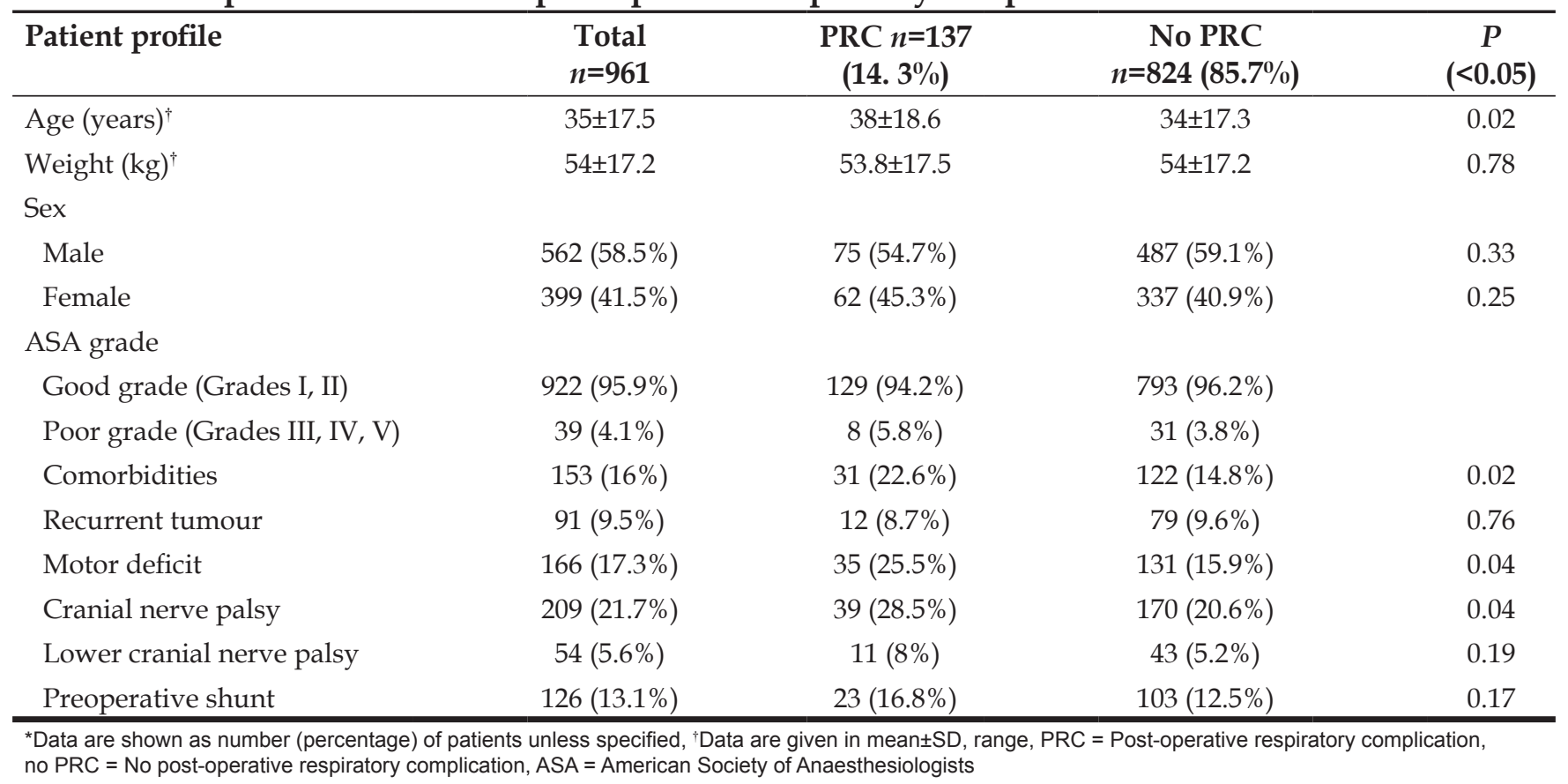

Table 3: Intraoperative factors and post-operative respiratory complications*

\begin{tabular}{|c|c|c|c|c|}
\hline Intraoperative factors & $\begin{array}{r}\text { Total } \\
n=961\end{array}$ & $\begin{array}{c}\text { PRC } \\
n=137(14.3 \%)\end{array}$ & $\begin{array}{c}\text { No PRC } \\
n=824(85.7 \%)\end{array}$ & $\begin{array}{c}P \\
(<0.05)\end{array}$ \\
\hline \multicolumn{5}{|l|}{ Anaesthetic technique } \\
\hline TIVA & $20(2 \%)$ & $3(2.2 \%)$ & $17(2.1 \%)$ & 0.17 \\
\hline Inhalational & $941(98 \%)$ & $134(97.8 \%)$ & $807(97.9 \%)$ & \\
\hline \multicolumn{5}{|l|}{ Position } \\
\hline Supine & $673(70 \%)$ & $88(64.2 \%)$ & $585(70.9 \%)$ & 0.005 \\
\hline Prone & $100(10 \%)$ & $15(10.9 \%)$ & $85(10.3 \%)$ & \\
\hline Lateral & $132(14 \%)$ & $17(12.4 \%)$ & $115(13.9 \%)$ & \\
\hline Sitting & $56(6 \% \%)$ & $17(12.4 \%)$ & $39(4.7 \%)$ & \\
\hline Hypertension & $33(3.4 \%)$ & $10(7.3 \%)$ & $23(2.8 \%)$ & 0.007 \\
\hline Hypotension & $139(14.5 \%)$ & $30(21.9 \%)$ & $109(13.2 \%)$ & 0.008 \\
\hline Tachycardia & $156(16.2 \%)$ & $37(27 \%)$ & $119(14.4 \%)$ & 0.00 \\
\hline Bradycardia & $31(3.2 \%)$ & $7(5.1 \%)$ & $24(2.9 \%)$ & 0.18 \\
\hline VAE & $12(1.2 \%)$ & $2(1.5 \%)$ & $10(1.2 \%)$ & 0.81 \\
\hline Brain bulge & $11(1 \%)$ & $2(1.5 \%)$ & $9(1.1 \%)$ & 0.71 \\
\hline Intraoperative blood loss $(\mathrm{ml})^{\dagger}$ & $742 \pm 784$ & $936 \pm 839$ & $710 \pm 770$ & 0.00 \\
\hline Intraoperative transfusion & $286(29.8 \%)$ & $113(82.5 \%)$ & $173(21 \%)$ & 0.00 \\
\hline Duration of surgery (min.) ${ }^{\dagger}$ & $289 \pm 272$ & $305 \pm 109$ & $286 \pm 290$ & 0.0006 \\
\hline
\end{tabular}

*Data are shown as number (percentage) of patients unless specified, ${ }^{\dagger}$ Data are given in mean $\pm S D$, range, $\mathrm{PRC}=\mathrm{Post}-\mathrm{operative}$ respiratory complication, no $\mathrm{PRC}=$ No post-operative respiratory complication, TIVA = Total intravenous anaesthesia, $\mathrm{VAE}=$ Venous air embolism

\section{DISCUSSION}

We conducted this prospective observational study to find out the incidences and nature of various respiratory complications in the early post-operative period and the associated risk factors in patients undergoing elective craniotomies. Instead of only pulmonary complications we studied the respiratory complications, which include the pulmonary complications as well the airway complications like airway obstruction and the need for intubation and mechanical ventilation. Airway complications can lead to significant impairment of 
Table 4: Post-operative factors and post-operative respiratory complications*

\begin{tabular}{lcccc}
\hline Post-operative & Total & PRC & No PRC & $\boldsymbol{P}$ \\
complications & $\boldsymbol{n = 9 6 1}$ & $\mathbf{n} \mathbf{1 3 7}(\mathbf{1 4 . 3} \%)$ & $\mathbf{n = 8 2 4} \mathbf{( 8 5 . 7 \% )}$ & $\mathbf{( < 0 . 0 5 )}$ \\
\hline Post-operative ventilation & $547(56.9 \%)$ & $118(86.1 \%)$ & $429(52.1 \%)$ & 0.003 \\
Post-operative haematoma & $29(3 \%)$ & $18(13.1 \%)$ & $11(1.3 \%)$ & 0.00 \\
Post-operative infarct & $60(6.2 \%)$ & $33(24.1 \%)$ & $27(3.3 \%)$ & 0.00 \\
Post-operative hydrocephalus & $22(2.3 \%)$ & $10(7.3 \%)$ & $12(1.5 \%)$ & 0.00 \\
Post-operative hypertension & $5(0.52 \%)$ & $0(0.00 \%)$ & $5(0.61 \%)$ & 0.36 \\
Post-operative hypotension & $3(0.31 \%)$ & $2(1.5 \%)$ & $1(0.12 \%)$ & 0.009 \\
GCS deterioration & $83(8.6 \%)$ & $68(49.6 \%)$ & $15(1.8 \%)$ & 0.00 \\
New motor deficits & $104(10.8 \%)$ & $43(31.4 \%)$ & $61(7.4 \%)$ & 0.00 \\
New cranial nerve palsies & $48(5 \%)$ & $11(8 \%)$ & $37(4.5 \%)$ & 0.08 \\
New lower cranial nerve palsies & $38(4 \%)$ & $15(11 \%)$ & $23(2.8 \%)$ & 0.00 \\
Post-operative seizures & $70(7.3 \%)$ & $24(17.5 \%)$ & $46(5.6 \%)$ & 0.00 \\
Mutism & $2(0.2 \%)$ & $0(0.00 \%)$ & $2(0.24 \%)$ & 0.56 \\
DI & $37(4 \%)$ & $12(8.8 \%)$ & $25(3.1 \%)$ & 0.001 \\
DVT & $7(0.72 \%)$ & $2(1.5 \%)$ & $5(0.61 \%)$ & 0.28 \\
Hypokalemia & $351(36.5 \%)$ & $112(81.8 \%)$ & $239(29 \%)$ & 0.00 \\
Hypernatremia & $43(4.5 \%)$ & $14(0.21 \%)$ & $29(3.51 \%)$ & 0.00 \\
Hyponatremia & $116(12.1 \%)$ & $39(28.5 \%)$ & $77(9.3 \%)$ & 0.00 \\
Fever & $135(14 \%)$ & $68(49.6 \%)$ & $67(8.1 \%)$ & 0.00 \\
Meningitis & $76(7.9 \%)$ & $35(25.5 \%)$ & $41(4.97 \%)$ & 0.00 \\
CSF leak & $11(1.1 \%)$ & $2(1.5 \%)$ & $9(1.1 \%)$ & 0.71 \\
Pseudomeningocoele & $5(0.52 \%)$ & $1(0.73 \%)$ & $4(0.5 \%)$ & 0.71 \\
Tension pneumocephalous & $6(0.62 \%)$ & $2(1.5 \%)$ & $4(0.5 \%)$ & 0.18 \\
\hline
\end{tabular}

*Data are shown as number (percentage), PRC = Post-operative respiratory complication, no PRC $=$ No post-operative respiratory complication,

GCS = Glasgow coma scale, DI = Diabetes insipidus, DVT = Deep venous thrombosis, CSF: Cerebrospinal fluid

Table 5: Incidence of post-operative respiratory complications

\begin{tabular}{lc}
\hline $\begin{array}{l}\text { Post-operative respiratory } \\
\text { complications }\end{array}$ & $\begin{array}{c}\text { Number } \\
\text { (percentage) } n=961\end{array}$ \\
\hline Respiratory complications (all types) & $137(14.3)$ \\
Purulent tracheobronchitis & $99(10.3)$ \\
Reintubation & $68(7.1)$ \\
Pneumonia & $40(4.1)$ \\
ARDS & $25(2.6)$ \\
Pneumothorax & $4(0.41)$ \\
Pulmonary oedema & $3(0.31)$ \\
\hline PRCs = Post-operative respiratory complications, ARDS = Acute respiratory \\
distress syndrome
\end{tabular}

pulmonary functions, hypoxia and hypercarbia and can affect the morbidity and mortality in these patients. We found that PRCs are common in patients undergoing elective craniotomies. The incidence of PRCs in our study was $14.3 \%$. The commonest respiratory complication was purulent tracheobronchitis which was found in $9.4 \%$ of patients. We found that various preoperative, intraoperative and post-operative factors were associated with development of PRC in these patients. We also found that occurrence of respiratory complications in the post-operative period is associated with poor neurological outcome at time of discharge.

A wide variation in the incidence of PRCs after neurosurgical procedures can be found in different studies. ${ }^{[1-3]}$ These large variations in the incidences of respiratory complications is due to differences in preoperative patients conditions, patient management, intraoperative complications, duration of post-operative follow-up, level of post-operative patient care and also because of different definitions used for defining these complications.

In a prospective study of 101 patients, Chari et al. observed respiratory complications only in $0.99 \%$ patients. ${ }^{[1]}$ The study sample included both intracranial as well as spinal surgery patients. Patients requiring direct admissions to an intensive care unit were excluded from the study and the patients were followed up only for the initial 4 hours after surgery. 
Bharati, et al.: Respiratory complications after craniotomies

Table 6: Difference of outcome between patients with and without post-operative respiratory complications*

\begin{tabular}{|c|c|c|c|c|}
\hline Outcome & Total $n=961$ & $\begin{array}{c}\text { PRC } \\
n=137(14.3 \%)\end{array}$ & $\begin{array}{c}\text { No PRC } \\
n=824(85.7 \%)\end{array}$ & $\begin{array}{c}P \\
(<0.05)\end{array}$ \\
\hline${\text { Hospital stay (days) }{ }^{\dagger}}^{\dagger}$ & $9.8 \pm 12.8(2-210)$ & $23.9 \pm 27.9$ & $7.5 \pm 4.7$ & 0.00 \\
\hline Mortality & $40(4.2 \%)$ & $32(23.3 \%)$ & $8(0.97 \%)$ & 0.00 \\
\hline \multicolumn{5}{|l|}{ Glasgow outcome score } \\
\hline Good outcome & $888(92.4)$ & $87(63.5 \%)$ & $801(97.2 \%)$ & 0.00 \\
\hline Poor outcome & $73(7.6)$ & $50(36.5 \%)$ & $23(2.8 \%)$ & \\
\hline
\end{tabular}

${ }^{*}$ Data are shown as number (percentage) of patients unless specified, ‘Data are given in mean \pm SD, range. PRC = Post-operative respiratory complication, no $\mathrm{PRC}=$ No post-operative respiratory complication

Table 7: Independent risk factors for occurrence post-operative respiratory complications after multivariate analysis

\begin{tabular}{lc}
\hline Variables & Odds ratio (95\% C.I.) \\
\hline Intraoperative tachycardia & $8.61(1.15-64.21)$ \\
Intraoperative blood transfusion & $5.62(2.93-10.80)$ \\
Post-operative GCS deterioration & $15.78(6.58-37.82)$ \\
Hypokalemia & $2.32(1.23-4.39)$ \\
Fever & $3.12(1.39-7.05)$ \\
\hline GCS = Glasgow coma scale, C.I = Confidence interval
\end{tabular}

Manninen et al. in a study of post-operative complications in 431 neurosurgical patients, observed PRCs in $2.8 \%$ of patients. ${ }^{[2]}$ The patients were observed only for the initial 4 hours and all kind of neurosurgical procedures were included in the study. Electively ventilated patients were excluded from data analysis. Respiratory complications after major intracranial procedures may be different from less invasive procedures like burr hole evacuation, ventriculostomy, omaya reservoir placements or spine surgeries.

To evaluate the incidence and risk factors of post-operative pulmonary complications, Sogame et al. did a prospective study in 236 patients who underwent elective intracranial procedures. ${ }^{[3]}$ The outcome of the study was the occurrence of post-operative pulmonary complications from immediate post-operative period until a new surgical intervention, hospital discharge or death. They found that post-operative pulmonary complications occurred in 58 patient $(24.6 \%)$ and 21 of these patients died. Various risk factors which were found significant in the multivariate analysis were infratentorial surgery, mechanical ventilation $>48$ hours, $>3$ days stay in the ICU, decreased level of consciousness in the post-operative period, $>300$ minutes of surgical duration and chronic lung diseases. In the multivariate analysis, we also found that deterioration in GCS was a significant risk factor for PRC.
In our study, the incidence of PRC was significantly increased in patients who received intraoperative blood transfusion. It was also found to be an independent risk factor for development of PRC. The incidence of PRC in patients who received blood transfusion was $82.5 \%$, whereas only $21 \%$ patients who did not receive blood transfusion developed PRC. Various studies have shown the association of higher incidence of post-operative infection in patients receiving homologous blood transfusion. ${ }^{[4-7]}$ The increased risk of infection after homologous blood transfusion is considered to be due to transfusion-induced immunosuppression. The probable mechanisms for the immunosuppression are reduced natural killer cell, decreased interleukin 2 productions, decreased CD4/CD8 ratio and decreased macrophage production. ${ }^{[8]}$

Respiratory complications were associated with the deterioration in consciousness in the post-operative period. In our series, the incidence of PRC was 49.5\% in patients who had GCS deterioration, whereas it was only $1.8 \%$ in patients who did not have deterioration of consciousness in the post-operative period. The chances of pulmonary aspiration, respiratory obstruction, hypoventilation and need for intubation and ventilation with subsequent complications increases following deterioration in the level of consciousness. ${ }^{[9-12]}$

We found hypokalemia as an independent risk factor for the concurrence of PRC. Hypokalemia was most probably due to decreased oral intake after surgery, use of cerebral decongestants (mannitol, furesemide) and hyperventilation associated with mechanical ventilation.

Hypokalemia in patients on mechanical ventilation may delay weaning due to respiratory muscle weakness and can predispose these patients to complications of prolonged intubation and mechanical ventilation.

Fever in the post-operative period was associated with increased incidence of PRC in our study. As rise in core body temperature is associated with increase in 
oxygen requirement and hyperventilation is normal physiological response to compensate this increased demand. Hyperventilation may lead to respiratory muscle fatigue, requirement for increased respiratory support and subsequent complications. It also prevents weaning of the patients from mechanical ventilation. The chance of pulmonary complications increases with the duration of mechanical ventilation. Reintubation after extubation may facilitate the development of nosocomial pneumonia because of alteration of glottic function and introduction of colonized oropharyngeal contents to lower airways. ${ }^{[13-15]}$

Intraoperative tachycardia has been shown to be associated with post-operative complications and poor outcome. ${ }^{[16]}$ In a retrospective review of 797 patients, who underwent elective surgical procedures, Reich et al. found that intraoperative tachycardia and hypertension had increased the post-operative mortality, prolonged the hospital stay with morbidity and were independent variables for poor post-operative outcome. ${ }^{[16]}$ In our series of neurosurgical patients, we found intraoperative tachycardia as an independent risk factor for development of PRCs. This association of intraoperative tachycardia with PRC could probably be due to presence of endotoxemia or exaggerated inflammatory response to surgery. ${ }^{[16]}$ The inflammatory response syndrome may lead to haemodynamic instability, respiratory insufficiency and multiorgan failure due to as diffuse microvascular thrombus in different organs, especially of the lungs. ${ }^{[17,18]}$

The prospective evaluation of a large sample of patients without many exclusion criteria representing a typical population of neurosurgical patients undergoing elective craniotomies very well reflects the incidence and nature of PRC in this group of patients. In this study, we have evaluated various factors not only in the preoperative and intraoperative period but also in the post-operative period to see their influence on the incidence of PRC. The use of a broader definition of respiratory complication, which included the pulmonary complications as well the airway complications like airway obstruction and the need for intubation and mechanical ventilation and also observation of patients for up to 72 hours, allowed us to include majority of early respiratory complications occurred in these patients. Since our study duration is limited to only 72 hours, we certainly have missed some of the respiratory complications beyond that period.

In conclusion, PRCs are common after elective intracranial procedures and are associated with increased ICU stay, hospital stay morbidity and mortality in these patients. Fever, hypokalemia, intraoperative blood transfusion and GCS deterioration are independent risk factors for the development of PRC. Hence, adequate control of fever, judicious use of blood transfusion, management of electrolyte imbalances and early intervention of airway following GCS deterioration should be done to reduce the incidence of PRC.

\section{REFERENCES}

1. Chari P, Bhardwaj N, Singh AR. Frequency of immediate postoperative complication in patients undergoing neurological procedures. J Anaesth Clin Pharmacol 2006;22:151-4.

2. Manninen PH, Raman SK, Boyle K, el-Beheiry H. Early postoperative complications following neurosurgical procedures. Can J Anaesth 1999;46:7-14.

3. Sogame LC, Vidotto MC, Jardim JR, Faresin SM. Incidence and risk factors for postoperative pulmonary complication in elective intracranial surgery. J Neurosurg 2008;109:222-7.

4. Nielsen HJ. Detrimental effects of perioperative blood transfusion. Br J Surg 1995;82:582-7.

5. Murphy PJ, Connery C, Hicks GL Jr, Blumberg N. Homologous blood transfusion as a risk factor for postoperative infection after coronary artery bypass graft operations. J Thorac Cardiovasc Surg 1992;104:1092-9.

6. Hill GE, Frawley WH, Griffith KE, Forestner JE, Minei JP. Allogeneic bloodtransfusion increases the risk of postoperative bacterial infection: A meta-analysis. J Trauma 2003;54:908-14.

7. Jensen LS, Andersen AJ, Christiansen PM, Hokland P, Juhl CO, Madsen G, et al. Postoperative infection and natural killer cell function following blood transfusion in patients undergoing elective colorectal surgery. Br J Surg 1992;79:513-6.

8. Kirkley SA. Proposed mechanisms of transfusion-induced immunomodulation. Clin Diagn Lab Immunol 1999;6:652-7.

9. Parr SM, Robinson BJ, Glover PW, Galletly DC. Level of consciousness on arrival in the recovery room and the development of early respiratory morbidity. Anaesth Intensive Care 1991;19:369-72.

10. Rodriguez JL, Gibbons KJ, Bitzer LG, Dechert RE, Steinberg SM, Flint LM. Pneumonia: Incidence, risk factors, and outcome in injured patients. J Trauma 1991;31:907-12.

11. Hsieh AH, Bishop MJ, Kubilis PS, Newell DW, Pierson DJ. Pneumonia following closed head injury. Am Rev Respir Dis 1992;146:290-4.

12. Chang SM, Parney IF, McDermott $M$, Barker FG $2^{\text {nd }}$, Schmidt MH, Huang W, et al. Perioperative complications and neurological outcomes of first and second craniotomies among patients enrolled in the Glioma Outcome Project. J Neurosurg 2003;98:1175-1.

13. Torres A, Gatell JM, Aznar E, el-Ebiary M, Puig de la Bellacasa J, González J, et al. Re-intubation increases the risk of nosocomial pneumonia in patients needing mechanical ventilation. Am J Respir Crit Care Med 1995;152:137-41.

14. Epstein SK, Ciubotaru RL. Independent effects of etiology of failure and time to reintubation on outcome for patients failing extubation. Am J Respir Crit Care Med 1998;158:489-93.

15. Torres A, Aznar R, Gatell JM, Jiménez P, González J, Ferrer A, et al. Incidence, risk, and prognosis factors of nosocomial pneumonia in mechanically ventilated patients. Am Rev Respir Dis 1990;142:523-8.

16. Reich DL, Bennett-Guerrero E, Bodian CA, Hossain S, Winfree $W$, Krol $M$. Intraoperative tachycardia and hypertension are independently associated with adverse outcome in noncardiac outcome surgery of long duration. Anesth Analg 2002;95:273-7.

17. Bennett-Guerrero E, Ayuso L, Hamilton-Davies C, White WD, Barclay GR, Smith PK, et al. Relationship of preoperative antiendotoxin core antibodies and adverse outcomes following cardiac surgery. JAMA 1997;277:646-50. 
18. Moretti EW, Newman MF, Muhlbaier LH, Whellan D, Petersen RP, Rossignol D, et al. Effects of decreased preoperative endotoxin core antibody levels on long-term mortality after coronary artery bypass graft surgery. Arch Surg 2006;141:637-41.
How to cite this article: Bharati SJ, Pandia MP, Rath GP, Bithal PK, Dash $\mathrm{HH}$. Respiratory complications in the early post-operative period following elective craniotomies. J Neuroanaesthesiol Crit Care 2015;2:114-20.

Source of Support: Nil, Conflict of Interest: None declared. 